\title{
Fatty Acids Profiling of Pigeon Squabs (Columba Livia Domestica) Using Gas-liquid Chromatography
}

\author{
Marwa Suliman Maged Ali, Heba Hussein Saleh Abdel-Naeem, Hayam Abd-Elaal Mansour, and Hamdy Mohamed \\ Bakry Abdelhady Zaki* \\ Department of Food Hygiene and Control, Faculty of Veterinary Medicine, Cairo University, Giza Square, Giza 12211, Egypt \\ *Corresponding author's Email: dvm.hamdy@gmail.com ORCID: 0000-0003-4571-2223
}

Received: 02 Apr. 2021 Accepted: 20 May 2021

\begin{abstract}
The available data from previous studies regarding the individual fatty acids profile of pigeon meat is limited. Therefore, the objective of the current study was to estimate the concentrations of different types of fatty acids in pigeon squabs meat. Seventy-five squabs samples were collected from butcher shops at Cairo and Giza governorates after that, the contained fat was extracted and subjected to fatty acid analysis using the gas-liquid chromatography technique. Results revealed that oleic acid had the highest percentage (36.61\%) followed by linoleic acid (17.79\%), palmitoleic fatty acid (8.95\%), and finally, linolenic fatty acid (4.46\%). On the other hand, low saturated fatty acids of pigeon meat were detected as palmitic and stearic fatty acids with percentages of $17.37 \%$ and $10.58 \%$, respectively. Moreover, a lowered trace of trans fatty acids was detected $(0.12 \%)$. Results indicated that pigeon meat could be considered as one of the beneficial meat sources due to its high content of both monosaturated fatty acid and polyunsaturated fatty acids as well as low saturated fatty acids content.
\end{abstract}

Keywords: Coronary heart diseases, Fatty acid profile, Gas-liquid Chromatography, Pigeon, Squab

\section{INTRODUCTION}

Pigeon meat has been consumed by rural populations of the world since old times and is recognized as delicious and nutritious poultry food. Recently, a growing interest in meat from alternative animal species like pigeons has been increased (Pomianowski et al., 2009; Ji et al., 2020). Besides that, the demand for functional food has greatly increased over the last decade with paying attention to the quality of consumed meat. The name of different pigeon breeds was mainly related to their origin which reveals how pigeons have been bred and kept around the world (Jerolmack, 2007). However, pigeon meats are not accepted as a food in some foreign cultures but they may be used as experimental animals. It is worthy to mention that not all species of pigeons raised in Egypt are accepted as food for example Columba Livia Sucumbery and Columba Livia Gadia are not used as food items. These two species differ in their ability to fly and their body characters, color, and beak types (Ramadan et al., 2011). Meanwhile, the meats of Columba Livia Domestica are acceptable as food by Egyptians with its many types, such as Balady, Romy, and Malty pigeons (Elsayed et al., 1980). Squabs is a term used to describe young pigeons, usually below four weeks of age (Abdel-Azeem, 2010; Mahdy, 2021).

Meat of pigeon squabs is characterized by high nutritional value, low-fat content, high protein content, high proportion of unsaturated fatty acids, less cholesterol value along with essential amino acids necessary for human growth. Moreover, it was considered a good source of different types of vitamins, including niacin, riboflavin, thiamine, B complex vitamins, and ascorbic acid, as well as essential minerals, including sodium, potassium, calcium, and iron (Paripuranam, 2014). Nowadays, consumption of heavy fat meat has become healththreatening and the use of these products has been gradually replaced by meat rich in protein, low in lipid, fine structured, easily digestible, such as pigeon meat (Gontariu and Buculei, 2009). Therefore, pigeon squabs are considered one of the best meats from the compositional point of view which is recommended for post-operatory or for clinical cases that require high protein level and low-fat diets (Buculei et al., 2010). Besides, this type of meat is beneficial for many consumers who began to consider the amount and type of fats in their diets due to many health concerns (Cooper and Horbanczuk, 2002), including the saturated and 
unsaturated fat content and their potential health effects especially for cardiovascular diseases (Simopoulos, 2008).

However, there is a paucity of research regarding the fatty acid profile of pigeon squabs in Egypt. Therefore, the current study was conducted to examine the fatty acid profiling of the Egyptian pigeon squabs.

\section{MATERIALS AND METHODS}

\section{Ethical approval}

This study design was approved by the Faculty of Veterinary Medicine, Cairo University according to the rules and guidelines of the ethical and animal welfare committee. In the current study, no live animal was used. All squabs meat samples used in this survey were purchased directly from local markets in form of chilled (4 ${ }^{\circ} \mathrm{C}$ ) carcass packages sold as edible food items on the shelf.

\section{Sample collection}

A total of 75 chilled $\left(4^{\circ} \mathrm{C}\right)$ carcasses of pigeon squabs (with the average carcass weight of 260-280g) were collected randomly from butcher shops located in Cairo and Giza governorates local markets. Samples were transferred immediately after purchasing in cooling icebox to the laboratory of the Food Hygiene and Control department and all samples were exposed to fatty acid profile analysis.

\section{Measurement of fatty acid profile}

The total lipids and fatty acids content of each squab meat sample were extracted according to Folch et al. (1957) and (Romero et al., 1998) then lipid extracts of each sample $(0.1 \mathrm{~g})$ were saponified in $100 \mu \mathrm{L}$ of $\mathrm{KOH}$ and methylated using boron trifluoride-methanol complex to achieve complete conversion to methyl esters (IUPAC, 1981). The fatty acid methyl esters were separated and analyzed by an automated gas-liquid chromatography (Model 6890 GC) equipped with a DB23 capillary column (Agilent Technology Inc.). Fatty acid peaks determined by gas chromatography were then used to calculate amounts of fatty acids according to calculations described by Slover and Lanza (1979).

\section{Statistical analysis}

All data were analyzed using IBM SPSS statistics 23 for windows using the descriptive statistics tool for each fatty acid. The Minimum value, Maximum value, and Mean value \pm Standard error of the mean was calculated for each fatty acid.

\section{RESULTS AND DISCUSSION}

Data about the fatty acid profile of peigon meat is very scarce, however, the fractionation of pigeon squabs meat fatty acids in the current study revealed that palmitic acid and stearic acid with a lipid number of C16:0 and C18:0 were the most predominating saturated fatty acids in peigon meat with percentages of 17.37 and 10.58, respectively (Table 1). Both palmitic acid and stearic acid are reasonable for the solid state of animal fat (Van Rooijen and Mensink, 2020). Generally, the percentage of saturated fats is recommended to be as low as possible in the human diet as they may lead to many coronary heart diseases and hypertension diseases (Fattore and Fanelli, 2013). Higher results of palmitic acid were obtained by Pomianowski et al. (2009) and Aydin (2005) with values of $22.87 \%$ and $19 \%$, respectively. However, a similar stearic acid value (10.63\%) was described by Pomianowski et al. (2009). Aydin (2005) reported the stearic acid value in pigeon breast muscle as $15.9 \%$.

Table 1. Saturated fatty acids profile of squab's meat samples $(\mathrm{n}=75)$

\begin{tabular}{lccc}
\hline SFA & Minimum & Maximum & Mean \pm SE \\
\hline C14:0 & 0.30 & 0.36 & $0.34 \pm 0.02 *$ \\
C15:0 & 0.02 & 0.06 & $0.04 \pm 0.01$ \\
C16:0 & 15.3 & 20.5 & $17.37 \pm 1.39$ \\
C17:0 & 0.10 & 0.15 & $0.12 \pm 0.01$ \\
C18:0 & 9.00 & 11.74 & $10.58 \pm 0.72$ \\
C20:0 & 0.09 & 0.14 & $0.11 \pm 0.01$ \\
C22:0 & 0.04 & 0.12 & $0.08 \pm 0.02$ \\
C24:0 & 0.33 & 0.54 & $0.41 \pm 0.06$ \\
\hline
\end{tabular}

*Data represent the percentage (\%) of every single fatty acid from the total fatty acid content; SFA: Saturated fatty acids; SE: Standard error of mean.

Among all obtained squabs fatty acids, oleic acid had the highest percentage (36.61\%, Table 2). Oleic acid is a monounsaturated fatty acid (containing one double bond) with a lipid number of C18:1. Oleic acid is known to have a major role in reducing many health risks as cardiovascular diseases (CVS) and thrombus formation. Moreover, oleic acid consumption may have a role in limiting saturated fat intake by the human body. Based on the level of dietary fat intake for both saturated and polyunsaturated fats, oleic acid intake is recommended to be not less than $10-15 \%$ for healthier diets to maintain good body functionality (Lopez-Huertas, 2010). The obtained results are in agreement with Pomianowski et al. (2009) who reported $37.42 \%$ for oleic fatty acid in Wrocławski pigeons. However, Aydin (2005) reported 
relatively lower oleic acid results of $27.6 \%$ for pigeon breast muscle fat.

Furthermore, the obtained fatty acids results in Table 2 showed that palmitoleic fatty acid had a relatively lower percentage of $8.95 \%$ than oleic acid with a lipid number of C16:1. Palmitoleic fatty acid also contains only one double bond within its chemical structure meaning that palmitoleic fatty acid is a monounsaturated fatty acid. Palmitoleic acid has major roles in metabolism inside the human body, including mediating cardiac growth, maintaining the integrity of endothelium, supporting the pancreatic $\beta$ cell functions, and counteracting the harmful effect of palmitic acid within the human body. Therefore, palmitoleic fatty acid helps reducing CVS diseases and diabetes by increasing the insulin sensitivity ( $\mathrm{Hu}$ et al., 2019). Nearly similar results of $7.56 \%$ for palmitoleic fatty acid were recorded by Pomianowski et al. (2009). On the other hand, Aydin (2005) obtained a lower result of 3.8\%.

Table 2. Monounsaturated fatty acids profile of squab's meat samples $(\mathrm{n}=75)$

\begin{tabular}{lccc}
\hline MUFA & Minimum & Maximum & Mean \pm SE \\
\hline $\mathrm{C} 14: 1$ & 0.12 & 0.15 & $0.13 \pm 0.01^{*}$ \\
$\mathrm{C} 15: 1$ & 0.23 & 0.30 & $0.27 \pm 0.02$ \\
$\mathrm{C} 16: 1$ & 7.9 & 10.00 & $8.95 \pm 0.54$ \\
$\mathrm{C} 17: 1$ & 0.05 & 0.10 & $0.07 \pm 0.01$ \\
$\mathrm{C} 18: 1$ & 35.00 & 37.50 & $36.61 \pm 0.70$ \\
$\mathrm{C} 20: 1$ & 0.30 & 0.38 & $0.33 \pm 0.02$ \\
$\mathrm{C} 24: 1$ & 0.14 & 0.24 & $0.18 \pm 0.03$
\end{tabular}

*Data represent the percentage (\%) of every single fatty acid from the total fatty acid content; MUFA: Monounsaturated fatty acids; SE: Standard error of mean.

Among the retrieved polyunsaturated fatty acids from pigeon squab's meat, linoleic acid had the largest share with a percentage of $17.79 \%$ (Table 3). Linoleic acid lipid number is C18:2 in the "Cis-" form and it contains two double bonds. Linoleic acid is known for its role in retarding the formation of atherosclerosis. Moreover, many organizations as Dietary Guidelines for America, American Heart Association, and WHO recommended the appropriate levels of linoleic acid as above $2 \%$ and up to $10 \%$ of the energy intake (Jandacek, 2017). A slightly lower value (15.96\%) was obtained by Pomianowski et al. (2009). In contrast, Aydin (2005) found a higher level (26.7\%) of linoleic acid content in pigeon breast muscle.

Additionally, $\alpha$-Linolenic fatty acid was the second fatty acid which had the major proportion in the polyunsaturated fatty acid content reaching $4.46 \%$ (Table
3). Generally, $\alpha$-Linolenic has a lipid number of C20:3n3 meaning that it is an omega 3 fatty acid (n3). As being an $\mathrm{n} 3$ fatty acid, $\alpha$-Linolenic is an essential fatty acid and cannot be synthesized inside the human body and required to be supplemented from the daily diets (De Seymour et al., 2019). The US National Institute of Health recommended a daily dietary intake of 1.1-1.6 g per day for $\alpha$-Linolenic fatty acid to decrease risks of CVS diseases, inflammation, cancer occurrence, Alzheimer's disease, macular degeneration, and rheumatoid arthritis (NIH, 2017).

Trans fatty acid found in pigeon meat (lipid number $=\mathrm{C} 18: 2 \mathrm{t} 2)$ indicated very low traces with $0.12 \%$ of the total obtained fatty acid content (Table 3). Additionally, other unknown traces of fatty acids $(0.10 \%)$ were recorded during the profiling of pigeon meat fat content.

Table 3. Polyunsaturated fatty acids profile of squab's meat samples $(\mathrm{n}=75)$

\begin{tabular}{lccc}
\hline PUFA & Minimum & Maximum & Mean \pm SE \\
\hline C18:2t2 & 0.10 & 0.16 & $0.12 \pm 0.02 *$ \\
C18:2 & 15.00 & 20.37 & $17.79 \pm 1.39$ \\
C18:3n6 & 0.06 & 0.11 & $0.08 \pm 0.01$ \\
C18:3n3 & 0.20 & 0.27 & $0.23 \pm 0.02$ \\
C20:2 & 0.24 & 0.31 & $0.27 \pm 0.02$ \\
C20:3n6 & 0.11 & 0.17 & $0.13 \pm 0.02$ \\
C20:4 & 0.15 & 0.22 & $0.18 \pm 0.02$ \\
C20:3n3 & 3.60 & 5.80 & $4.46 \pm 0.59$ \\
C22:3 & 0.22 & 0.38 & $0.32 \pm 0.04$ \\
C22:4 & 0.40 & 0.66 & $0.53 \pm 0.07$ \\
C22:5 & 0.12 & 0.19 & $0.15 \pm 0.02$ \\
\hline
\end{tabular}

*Data represent the percentage (\%) of every single fatty acid from the total fatty acid content; PUFA: Polyunsaturated fatty acids; SE: Standard error of mean.

\section{CONCLUSION}

Besides being an excellent source of protein, pigeon meat is considered a healthy fat source. It contains high amounts of oleic fatty acid and a relatively low percentage of palmitic and stearic saturated fatty acids which could assist in controlling and preventing many cardiovascular diseases. Also, from the nutritional aspect, pigeon meat contains a little trace of trans fatty acids which favors its consumption as a source of nutrition. Future studies need to reveal the effect of various feeding and husbandry methods on the pigeon squabs fatty acid profile. Also, the relationship between fatty acid profile and other meat quality parameters needs to be fully studied for a proper type of pigeon squabs meat quality. 


\section{DECLARATION}

\section{Competing interests}

The authors declare that there is no conflict of interest

\section{Authors' contribution} this study

All authors shared the same effort during performing

\section{Ethical considerations}

All authors approved the manuscript submission and its content. Ethical issues (including plagiarism, consent to publish, misconduct, data fabrication and/or falsification, double publication and/or submission, and redundancy) have been checked by the authors.

\section{REFERENCES}

Abdel-Azeem FA (2010). The composition of the crop milk in Egyptian Baladi pigeons and its role in growth of squabs. Egyptian Poultry Science Journal, 30(4): 1003-1015. Available at: http://www.epsaegypt.com/pdf/2010_december/6-1227.pdf

Aydin R (2005). The effect of conjugated linoleic acid on the fatty acid composition of different tissues and yolk lipids in pigeons. South African Journal of Animal Science, 35(4): 253-260.

DOI: https://www.doi.org/10.4314/sajas.v35i4.3968

Buculei A, Gontariu I, and Rebenciuc I (2010). Comparative study regarding the aging influence upon the quality of pigeon and turkey meat. Scientific Papers, 53: 485-490. Available at: http://www.uaiasi.ro/zootehnie/Pdf/Pdf_Vol_53/Amelia_Bu culei.pdf

Cooper RG, and Horbanczuk JO (2002). Anatomical and physiological characteristics of ostrich (Struthio camelus var. domesticus) meat determine its nutritional importance for man. Animal Science Journal, 73(3): 167-173. DOI: https://www.doi.org/10.1046/j.1344-3941.2002.00024.X

De Seymour JV, Simmonds LA, Gould J, Makrides M, and Middleton P (2019). Omega-3 fatty acids to prevent preterm birth: Australian pregnant women's preterm birth awareness and intentions to increase omega-3 fatty acid intake. Nutrition Journal, 18(1): 1-6. DOI: https://www.doi.org/10.1186/s12937-019-0499-2

Elsayed WA, Shehab AH, Mourad FE, El- Nahry FI, and Said AK (1980). Biochemical and biological evaluation of pigeon meats: Effect of type, age and sex. Nahrung, 24(9): 821-828. DOI: https://www.doi.org/10.1002/food.19800240903

Fattore E, and Fanelli R (2013). Palm oil and palmitic acid: A review on cardiovascular effects and carcinogenicity. International Journal of Food Sciences and Nutrition, 64(5): 648-659.

DOI: https://www.doi.org/10.3109/09637486.2013.768213

Folch J, Lees M, and Sloane-Stanley GHS (1957). A simple method of the isolation and purification of total lipids from animal tissues. Journal of Biochemical Chemistry, 226:
497-509. DOI: https://www.doi.org/10.1016/s00219258(18)64849-5

Gontariu I, and Buculei A (2009). A study on the influence of ageing upon the quality of the pigeon meat. Journal of Agroalimentary Processes and Technologies, 15(3): 421425. Available at: https://www.journal-ofagroalimentary.ro/admin/articole/95158L78_Buculei_Ameli a_421-425.pdf

Hu W, Fitzgerald M, Topp B, Alam M, and O'Hare TJ (2019). A review of biological functions, health benefits, and possible de novo biosynthetic pathway of palmitoleic acid in macadamia nuts. Journal of Functional Foods, 62: Article ID $103520 . \quad$ DOI: https://www.doi.org/10.1016/j.jff.2019.103520

International Union of Pure and Applied Chemistry (IUPAC) (1981). Standard methods for the analysis of oils, Fats and Derivatives, $6^{\text {th }}$ Edition, ed. C. Paquot, Published by International Union of Pure and Applied Chemistry, Oxford, Great Britain. 233-246. DOI: https://doi.org/10.1351/pac198254010233

Jandacek RJ (2017). Linoleic acid: A nutritional quandary. Healthcare, 5(2): 25-33. https://www.doi.org/10.3390/healthcare5020025

Jerolmack C (2007). Animal archeology: Domestic pigeons and the nature-culture dialectic. Qualitative Sociology Review, 3(1): 74-95. Available at: http://citeseerx.ist.psu.edu/viewdoc/download?doi=10.1.1.1 $079.8269 \&$ rep $=$ rep $1 \&$ type $=$ pdf

Ji F, Zhang D, Shao Y, Yu X, Liu X, Shan D, and Wang Z (2020). Changes in the diversity and composition of gut microbiota in pigeon squabs infected with Trichomonas gallinae. Scientific Reports, 10(1): Article number 19978. DOI: https://www.doi.org/10.1038/s41598-020-76821-9

Lopez-Huertas E (2010). Health effects of oleic acid and long chain omega-3 fatty acids (EPA and DHA) enriched milks. A review of intervention studies. Pharmacological Research, 61(3): 200-207. DOI: https://www.doi.org/10.1016/j.phrs.2009.10.007

Mahdy MA (2021). Comparative morphological study of the oropharyngeal floor of squabs and adult domestic pigeons (Columba livia domestica). Microscopy Research and Technique, $84(3)$ : 499-511. DOI: https://www.doi.org/10.1002/jemt.23606

National Institutes of Health (NIH) (2017). Omega-3 fatty acids - health professional fact sheet. US National Institutes of Health, Office of Dietary Supplements. Available at: https://ods.od.nih.gov/factsheets/Omega3FattyAcidsHealthProfessional/\#h2

Paripuranam D (2014). Proximate, physical and mineral compositions of pigeon meal used as fish bait. Journal of Chemical and Pharmaceutical Research, 6(1): 669-673. Available at: http://jocpr.com/vol6-iss1-2014/JCPR-2014-61-669-673.pdf

Pomianowski JF, Mikulski D, Pudyszak K, Cooper RG, Angowski M, Jóźwik A, and Horbańczuk JO (2009). Chemical composition, cholesterol content, and fatty acid profile of pigeon meat as influenced by meat-type breeds. Poultry Science, 88(6): 1306-1309. DOI: https://www.doi.org/10.3382/ps.2008-00217 
Ramadan S, Abe H, Hayano A, Yamaura J, Onoda T, Miyake T, and Inoue-Murayama M (2011). Analysis of Genetic Diversity of Egyptian Pigeon Breeds. The Journal of Poultry Science, $\quad 48(2)$ : 79-84. DOI: https://www.doi.org/10.2141/jpsa.010109

Romero A, Cuesta C, and Sanchez-Muniz FJ (1998). Effect of oil replenishment during deep fat frying of frozen foods in sunflower oil and high-oleic acid sunflower oil. American Oil Chemist's Society, 75: 161-167. DOI: https://www.doi.org/10.1007/s11746-998-0028-5

Simopoulos AP (2008). The importance of the omega-6/omega-3 fatty acid ratio in cardiovascular disease and other chronic diseases. Experimental Biology and Medicine (Maywood). 233(6): 674-688. DOI: https://www.doi.org/10.3181/0711MR-311

Slover HT, and Lanza E (1979). Quantitative analysis of food fatty acids by capillary gas chromatography. American Oil Chemist's Society, 56: 933-934. DOI: https://www.doi.org/10.1007/bf02674138

Van Rooijen MA, and Mensink RP (2020). Palmitic Acid Versus Stearic Acid: Effects of Interesterification and Intakes on Cardiometabolic Risk Markers-A Systematic Review. Nutrients, $12(3)$ : 615-639. https://www.doi.org/10.3390/nu12030615 